Artículo

\title{
Evaluación y predicción de la infiltración en un Andosol bajo diferentes usos de suelo
}

\author{
Silvia Janeth Béjar-Pulido \\ Israel Cantú-Silva ${ }^{\S}$ \\ María Inés Yáñez-Díaz \\ Erik Orlando Luna-Robles
}

Facultad de Ciencias Forestales-Universidad Autónoma de Nuevo León. Carretera Nacional núm. 85 km 145, Linares, Nuevo León, México. CP. 67700. Tel. 821 2124895. (chivisjan@hotmail.com; inesyd@gmail.com; meranroka@hotmail.com).

${ }^{\S}$ Autor para correspondencia: icantu59@gmail.com.

\section{Resumen}

La infiltración es un indicador de la capacidad del suelo para retener o drenar el agua, se ve influenciada por la cubierta vegetal y tipo de suelo, que al ser alterada modifica sus atributos. El objetivo de la investigación fue evaluar el comportamiento de la infiltración en un Andosol. Se establecieron parcelas de $100 \mathrm{~m}^{2}$, en cuatro áreas bajo dos usos de suelo: un área forestal y tres agrícolas [dos parcelas de cultivo de aguacate (bajo manejo orgánico y convencional) y una parcela más de macadamia con manejo orgánico], el año de referencia de la investigación fue 2018. Se determinó la infiltración in situ por medio del método de doble anillo tomando lecturas por un período de 150 minutos con recargas variables. Se obtuvieron los parámetros de tres modelos empíricos para estimar la infiltración: Horton, Kostiakov y Mezencev. El uso forestal presentó la mayor infiltración inicial, final, básica y acumulada observadas con valores de 1 880, 863.47, $885.92 \mathrm{~mm} \mathrm{~h}^{-1}$ y $2793.29 \mathrm{~mm}$, mientras que la parcela de aguacate con manejo convencional mostro los valores más bajos con $620,248.27,254.83 \mathrm{~mm} \mathrm{~h}^{-1}$ y $872.49 \mathrm{~mm}$, respectivamente. Los resultados del ajuste de modelos demostraron que el modelo de Kostiakov predice en mejor medida el proceso de infiltración, esto de acuerdo con los valores de NSE, r, MAE y R ${ }^{2}$. Los cambios de uso forestal a usos agrícolas provocan cambios en las variables hidrológicas del recurso suelo. La infiltración presentó mayores tasas de velocidad bajo manejos orgánicos que convencionales.

Palabras clave: aguacate, cambio uso del suelo, bosque pino-encino, macadamia.

Recibido: julio de 2021

Aceptado: octubre de 2021 


\section{Introducción}

Las primeras etapas de establecimiento de cultivos de aguacate en el estado de Michoacán fueron sobre zonas de agricultura de temporal y en menor medida sobre forestal (Burgos et al., 2012); sin embargo, durante los últimos 15 años la conversión de áreas forestales a cultivos de aguacate ha ido acelerándose significativamente, atribuido principalmente a la rentabilidad de este (Chávez et al., 2012).

Específicamente, la vegetación y el suelo son dos aspectos estructurales sensibles ante los cambios o disturbios en los ecosistemas, usualmente el ciclo hidrológico es uno de los principales procesos afectados lo cual provoca cambios en la disponibilidad de agua del ecosistema (Galicia, 2014). De acuerdo con Regüés et al. (2012), los cambios de uso de suelo provocan principalmente cambios a corto plazo en las variables hidrológicas del recurso suelo. La infiltración del agua en el suelo juega un papel fundamental en el ciclo hidrológico. Dependiendo de las condiciones geológicas y ecológicas, puede permanecer en el suelo en forma de humedad, escurrir como flujo subsuperficial y aflorar como una naciente efímera; o recargar el acuífero, entre otros (Batres y Barahona, 2016).

En el control de la tasa y capacidad de infiltración desempeñan un papel importante la textura y estructura del suelo, los tipos de vegetación, el contenido de agua del suelo, la temperatura del suelo y la intensidad de precipitación (Rivera y Dallatorre, 2018). En México el empleo de modelos matemáticos para determinar la tasa de infiltración ha sido muy poco estudiado, la mayoría han tenido éxito, dependiendo del tipo de modelo y características de suelo a evaluar (Green y Ampt, 1911; Singh et al., 2018). Mishra et al. (2003) indican que para la predicción de la infiltración se han creado distintas ecuaciones, las cuales, se pueden clasificar en tres tipos de modelos 1) físicos, se deducen de la ley de conservación de masas y la ley de Darcy; 2) semiempíricos; consisten en hipótesis simples sobre la tasa de infiltración y la relación de infiltración; y 3) empíricos, se basan en datos de campo y experimentos de laboratorio.

Siendo estos últimos los considerados para la modelación de infiltración sobre el suelo tipo Andosol. El objetivo del presente estudio fue predecir el proceso de infiltración a partir de datos obtenidos en campo con la metodología del infiltrómetro de doble anillo, en cuatro áreas bajo dos usos de suelo: un área con vocación forestal y dos cultivos agrícolas bajo dos tipos de manejo en un suelo tipo Andosol en Uruapan, Michoacán. Con base en los datos observados se procedió a evaluar y comparar la bondad de ajuste de tres modelos empíricos para la estimación de la infiltración (Horton, Kostiakov y Mezencev), mediante indicadores estadísticos.

\section{Materiales y métodos}

\section{Área de estudio}

La investigación se llevó a cabo en el Ejido de Toreo El Alto en el municipio de Uruapan, Michoacán, ubicado en la parte sur de la Sierra Purépecha, comprendido entre la latitud norte $19^{\circ}$ 28 ' 22.2' ' y longitud oeste 102 $00^{\circ}$ ' 19.7'. Se encuentra en el Eje Neovolcánico Transversal a una altitud de $1890 \mathrm{msnm}$ y pertenece a la región del Río Balsas. El clima en la zona es templado semicálido húmedo, con abundantes lluvias en verano y un porcentaje de lluvia invernal menor al 5\% (A)C $(\mathrm{m})(\mathrm{w})$ (García, 1981), con una temperatura media anual de $16{ }^{\circ} \mathrm{C}$ y registra una precipitación media anual de $1500 \mathrm{~mm}$. El suelo dominante es Andosol, particularmente, presentan baja densidad aparente y alta capacidad de retención de agua (Alcalá et al., 2001). 


\section{Diseño experimental}

Se establecieron cuatro parcelas de $100 \mathrm{~m}^{2}$ bajo dos usos de suelo: un área con vocación forestal y dos agrícolas (dos parcelas de cultivo de aguacate [bajo manejo orgánico y convencional] y una parcela más de plantación de macadamia con manejo orgánico). Los cuales se describen a continuación: a) forestal: de acuerdo con la clasificación de Rzedowski (2006) el tipo de vegetación es un bosque de coníferas representado por una mezcla de especies del género Pinus y Quercus, entre ellos destacan: Pinus devoniana Lindley, Pinus pseudostrobus Lindl, Pinus lawsonii Roezl, Pinus leiophylla Schl. \& Cham, Quercus rugosa Neé, Quercus laurina Humb et Bonpl, Arbustus xalapensis Kunth y Fraxinus udheii (Wenz) Lingelsh este corresponde a un bosque no coetáneo sin manejo, con una densidad de 600 árboles por hectárea; y b) agrícola: se evaluaron dos tipos de cultivos, dos huertas de aguacate (Persea americana Mill var. Hass) con manejo convencional y orgánico y una huerta de macadamia orgánica (Macadamia integrifolia Maiden \& Betche). Las plantaciones tienen una edad promedio de 60 años, aunque existen individuos de 5-10 años posteriores al manejo de sanidad en las áreas. La plantación usada para las tres parcelas agrícolas fue de tipo marco real $(10 \times 10)$ con una densidad de 100 árboles por hectárea y una producción promedio de $15,6.25$ y $3.2 \mathrm{t} \mathrm{ha}^{-1}$, respectivamente.

El manejo orgánico no implica el uso de químicos (fertilizantes y pesticidas) y solo utiliza composta de bovino, con una dosis de $25 \mathrm{~kg}$ por árbol $\left(2500 \mathrm{~kg} \mathrm{ha}^{-1}\right)$ colocados a media sombra cada tres años. Mientras que el manejo convencional contempla el uso de pesticidas y prácticas como podas, faenas y fertilización química (sulfato de cobre pentahidratado $\left[\mathrm{CuSO}_{4.5} \mathrm{H}_{2} \mathrm{O}\right.$ ] y Urea [CO $\left(\mathrm{NH}_{2}\right)_{2}$ ] con fórmula de aplicación foliar 20N-30P-10K, complementada con macro y micronutrientes (S-1.6\%, Mg-0.8\%, Fe-0.05\%, Cu, Mn, B, Zn y Mo al 0.01\%). Algunas características importantes del suelo Andosol que influyen en los procesos hidrológicos se presentan en la (Cuadro 1).

Cuadro 1. Valores promedio de las características del suelo Andosol en las parcelas evaluadas.

\begin{tabular}{cccc}
\hline Parcelas & $\mathrm{MO} \%$ & $\mathrm{DA}\left(\mathrm{g} \mathrm{cm}^{-3}\right)$ & Clase Textural \\
\hline Forestal & $13.02 \pm 1.94$ & $0.56 \pm 0.1$ & $\mathrm{CA}$ \\
Aguacate convencional & $16.41 \pm 1.32$ & $0.57 \pm 0.05$ & $\mathrm{CL}$ \\
Aguacate orgánico & $9.05 \pm 1.2$ & $0.82 \pm 0.12$ & $\mathrm{CA}$ \\
Macadamia & $13.8 \pm 2.32$ & $0.69 \pm 0.06$ & $\mathrm{CA}$ \\
\hline
\end{tabular}

$\mathrm{MO} \%=$ porcentaje de materia orgánica; $\mathrm{DA}=$ densidad aparente, clase textural: $\mathrm{CA}=$ franco arenoso; $\mathrm{CL}=$ franco limoso (Béjar et al., 2020).

Específicamente, se efectuaron tres repeticiones en cada parcela para medir la infiltración en campo por el método de doble anillo; cabe señalar, que otros estudios han empleado un número similar de repeticiones (Landini et al., 2007; Tejedor, Neris y Jiménez, 2013; Weber y Apestegui, 2016; Luna et al., 2020). Las pruebas de infiltración se realizaron debajo del dosel a una distancia mínima de $5 \mathrm{~m}$ del tallo. El tamaño del anillo interno fue de $15 \mathrm{~cm}$ de diámetro y $25 \mathrm{~cm}$ de altura y el externo de $28 \mathrm{~cm}$ de diámetro por $30 \mathrm{~cm}$ de altura, los anillos fueron insertado en el suelo a una profundidad de $5 \mathrm{~cm}$. 
Iniciada la prueba se hicieron registros $(\mathrm{en} \mathrm{cm}$ ) por minuto durante cinco minutos, cada cinco minutos hasta completar la primera hora de evaluación, en la segunda hora, las mediciones se efectuaron cada 10 min para finalizar dos lecturas cada 15 min, para un total de $150 \mathrm{~min}(2.5 \mathrm{~h})$. Se usó una lámina inicial de $28 \mathrm{~cm}$, cuando el nivel del agua llegaba a $8 \mathrm{~cm}$, se recargaba el cilindro interior, depositando el agua lentamente, el cilindro exterior se rellenaba cuando disminuía su nivel. La medición se realizó durante el periodo de lluvias en el mes de junio de 2018, presentándose promedios de la humedad del suelo de $60.6 \%$.

\section{Velocidad de infiltración}

La velocidad de infiltración es la capacidad con la cual el agua penetra en el suelo. Generalmente se mide con base en la profundidad (en $\mathrm{mm}$ ) de la lámina de agua que logra penetrar en el suelo en una hora (Castellanos-Navarrete et al., 2013). Para determinar la tasa de infiltración se empleó la siguiente expresión (Zhang et al., 2017; Yáñez-Díaz et al., 2019): $\mathrm{f}(\mathrm{t})=\frac{\Delta \mathrm{I}}{\Delta \mathrm{t}} * 600$. Donde: $\mathrm{f}(\mathrm{t})$ es la tasa de infiltración $\left(\mathrm{mm} \mathrm{h}^{-1}\right) ; \Delta \mathrm{I}$ es la infiltración $(\mathrm{cm}) ; \Delta$ t es el tiempo durante el periodo de infiltración (min); y 600 es un coeficiente de conversión a $\mathrm{mm} \mathrm{h}^{-1}$.

\section{Infiltración acumulada}

Considera la sumatoria de los valores puntuales de infiltración obtenidos de la curva de velocidad de infiltración instantánea. La ecuación de la infiltración acumulada se obtiene determinando el área formada entre dicha curva y los ejes, es decir integrando su ecuación entre los valores límites: $t_{\text {inicial }}=0$ y el $t_{\text {final }}$ (Delgadillo y Pérez, 2016).

\section{Infiltración básica}

Inicialmente, al aplicar agua, el valor de infiltración es alto y a medida que se incrementa el contenido de agua en el suelo, disminuye paulatinamente hasta llegar a un valor constante denominado velocidad de infiltración básica. La velocidad de la infiltración del agua en el suelo, en el momento en que la variación de ésta con respecto al tiempo es muy lenta y constante. Generalmente, esta condición de la infiltración se consigue cuando el suelo ha alcanzado su capacidad de campo (Delgadillo y Pérez, 2016). La infiltración básica se determinó a partir de los promedios de las últimas tres lecturas $(120,135$ y $150 \mathrm{~min})$ de la prueba de infiltración (YáñezDíaz et al., 2019).

\section{Modelos de infiltración}

Tras determinar la tasa de infiltración en campo (infiltración real) se realizó el ajuste de tres modelos empíricos: Horton, Kostiakov y Mezencev, con los datos obtenidos (Cuadro 2). Para determinar los valores de los parámetros de los modelos ajustados se utilizó el complemento Solver de Microsoft Excel 2007 y por medio del método de mínimos cuadrados se calcularon los valores predictivos de la infiltración. 
Cuadro 2. Modelos empíricos empleados para la determinación de la tasa de infiltración.

\begin{tabular}{lll}
\hline Modelo & Ecuación & Definición \\
\hline Horton (1939) & $\mathrm{f}(\mathrm{t})=\mathrm{f}_{\mathrm{b}}+\left(\mathrm{f}_{0}-\mathrm{f}_{\mathrm{b}}\right)^{\mathrm{e}^{\mathrm{k}} \mathrm{kt}}$ & $\begin{array}{l}\mathrm{f}(\mathrm{t})=\text { tasa de infiltración en el instante, } \mathrm{t}, \mathrm{f}_{\mathrm{b}}, \mathrm{f}_{0} \text { y K son } \\
\text { parámetros determinados por el modelo, en el cual } \mathrm{f}_{\mathrm{b}} \text { es } \\
\text { considerado como capacidad de infiltración }\end{array}$ \\
$\begin{array}{l}\text { Kostiakov (Weber } \\
\begin{array}{l}\text { y Apestegui, 2013) } \\
\text { Mezencev (Weber }\end{array}\end{array} \quad \mathrm{f}(\mathrm{t})=\mathrm{at}^{-\mathrm{b}}$ & $\begin{array}{l}\text { a y b son los parámetros determinados del modelo, } \\
\text { mientras t es el tiempo }\end{array}$ \\
\hline
\end{tabular}

Además de la tasa de infiltración, también se obtuvieron la infiltración inicial (Fi), final (Ff), básica $(\mathrm{Fb})$ y acumulada $(\mathrm{Fac})$. La Fi $\left(\mathrm{mm} \mathrm{h}^{-1}\right)$ corresponde la tasa de infiltración en el tiempo 1, Ff (mm $\mathrm{h}^{-1}$ ) a la tasa de infiltración del minuto 150 , la $\mathrm{Fb}\left(\mathrm{mm} \mathrm{h}^{-1}\right)$ fue determinada con el promedio de las últimas tres lecturas de la tasa de infiltración y la Fac $(\mathrm{mm})$ se calculó con la sumatoria del volumen de agua infiltrado del tiempo 1 más agua infiltrada en el tiempo 2 hasta llegar al tiempo n.

\section{Comparación y validación de los modelos}

Los estadísticos para la comparación y validación de los modelos fueron el coeficiente de eficiencia del modelo Nash Sutcliffe (NSE), coeficiente de Pearson (r) y error absoluto medio (MAE). Y se expresan de la siguiente manera: Coeficiente de eficiencia del modelo Nash Sutcliffe (NSE): $\mathrm{NSE}=1-\frac{\sum_{\mathrm{i}=1}^{\mathrm{n}}\left(\mathrm{Obs}_{\mathrm{i}}-\mathrm{SIM}_{\mathrm{i}}\right)^{2}}{\sum_{\mathrm{i}=1}^{\mathrm{n}}\left(\mathrm{Obs}_{\mathrm{i}}-\overline{\mathrm{Obs}}\right)^{2}}$, dónde: $\mathrm{Obs}_{\mathrm{i}}=$ datos de observación, $\mathrm{SIM}_{\mathrm{i}}=$ representa el valor de simulación/modelo y $\overline{\mathrm{Obs}}=$ valor medio de la observación. Coeficiente de Pearson $(\mathrm{r}): \mathrm{r}=$

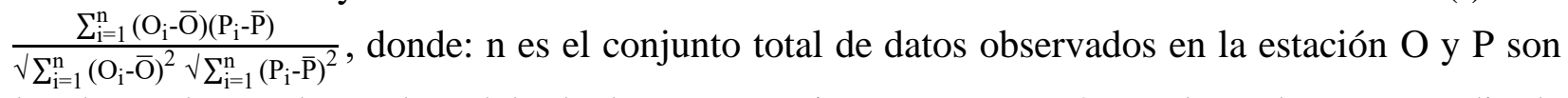
los datos observados y el modelo de datos, respectivamente y $\mathrm{P}_{\mathrm{i}}$ y $\mathrm{O}_{\mathrm{i}}$ son los valores promedio de los datos del modelo y los datos observados, respectivamente. Error absoluto medio (MAE): $\mathrm{MAE}=\frac{1}{\mathrm{n}} * \sum_{\mathrm{i}=1}^{\mathrm{n}}\left|\mathrm{O}_{\mathrm{i}}-\mathrm{P}_{\mathrm{i}}\right|$. Donde: $\mathrm{n}=$ el número de errores, $\left|\mathrm{O}_{\mathrm{i}}-\mathrm{P}_{\mathrm{i}}\right|=$ errores absolutos.

\section{Análisis estadísticos}

A los datos obtenidos de campo (Fi, Ff, Fb y Fac) se les aplicó una prueba normalidad (ShapiroWilk) y homogeneidad de varianza (Levene), cumpliendo con los supuestos se realizó un análisis de varianza de un factor (Anova) y posteriormente una comparación de medias (Tukey $p \leq 0.05$ ) para determinar diferencias significativas de infiltración entre los distintos usos de suelo. Todos los análisis estadísticos se realizaron utilizando la versión 13.0 del software SPSS (SPSS Inc., Chicago, IL), con un nivel de confianza de $p \leq 0.05$.

\section{Resultados y discusión}

\section{Infiltración en campo}

De acuerdo con el Anova entre usos de suelo, se presentaron diferencias significativas $(p \leq 0.05)$ para las variables Fi, Ff, Fb y Fac con un nivel de significancia de 0.003, 0.000112, 0.000138 y 0.001 , respectivamente. Las variables fueron analizadas por una prueba de Tukey $(p \leq 0.05)$ para 
describir su comportamiento, donde aguacate convencional mostró una disminución de 67, 71, 71 y $68 \%$ en la infiltración inicial, final, básica y acumulada, respecto al uso forestal que presentó los valores más altos de las variables hidrológicos evaluados (Cuadro 3).

Cuadro 3. Valores medios de las variables hidrológicas en las parcelas evaluadas.

\begin{tabular}{ccccc}
\hline \multirow{2}{*}{ Parcelas } & \multicolumn{4}{c}{ Variables hidrológicas } \\
\cline { 2 - 5 } & $\mathrm{Fi}\left(\mathrm{mm} \mathrm{h}^{-1}\right)$ & $\mathrm{Ff}\left(\mathrm{mm} \mathrm{h}^{-1}\right)$ & $\mathrm{Fb}\left(\mathrm{mm} \mathrm{h}^{-1}\right)$ & $\mathrm{Fac}(\mathrm{mm})$ \\
\hline Forestal & $1880 \mathrm{a}$ & $863.47 \mathrm{a}$ & $885.92 \mathrm{a}$ & $2793.29 \mathrm{a}$ \\
Aguacate convencional & $620 \mathrm{~b}$ & $248.27 \mathrm{~b}$ & $254.83 \mathrm{~b}$ & $872.49 \mathrm{~b}$ \\
Aguacate orgánico & $800 \mathrm{bc}$ & $404.4 \mathrm{bc}$ & $413.63 \mathrm{bc}$ & $1242.26 \mathrm{bc}$ \\
Macadamia & $1460 \mathrm{ac}$ & $506.53 \mathrm{c}$ & $519.46 \mathrm{c}$ & $1756.72 \mathrm{ac}$ \\
\hline
\end{tabular}

$\mathrm{Fi}=$ infiltración inicial; $\mathrm{Ff}=$ infiltración final; $\mathrm{Fb}=$ infiltración básica; $\mathrm{Fac}=$ infiltración acumulada. Letras distintas representan diferencias significativas (Tukey $p \leq 0.05$ ).

Los resultados presentan variaciones significativas entre los distintos usos de suelo, particularmente se observan descensos de infiltración del uso agrícola respecto al forestal, Florez et al. (2009) encontraron tendencias similares evaluando el comportamiento de la infiltración bajo sistemas agrícolas, pecuarios y vegetación nativa, definiendo que los cambios de uso de suelo afectan a corto plazo a las variables físicas e hidrológicas del recurso suelo.

De acuerdo con los resultados el uso de agrícola con manejo convencional presentó los valores más bajos de infiltración (Cuadro 3), pudiendo ser atribuido al empleo de las distintas practicas mecanizadas (podas de saneamiento, colecta de fruta y fertilizaciones mecanizadas), las cuales aumentan la densidad aparente en la parte superficial del suelo reduciendo el tamaño de poros y con ello provocando efectos en la infiltración, y otros procesos como pérdidas de suelo por escorrentía (Bravo-Espinoza et al., 2014).

Los resultados de la infiltración inicial de la presente investigación son similares a los obtenidos por García-Hernández et al. (2008), quienes encontraron tasas de infiltración en bosque de pino y bosque encino de 1920 y $1320 \mathrm{~mm} \mathrm{~h}^{-1}$, respectivamente, en la Sierra Gorda de Querétaro, señalando que la velocidad con la que el agua es infiltrada en el suelo varía de acuerdo a las condiciones del suelo y cubierta vegetal, por lo que cambios de uso de suelo pueden tener un impacto más significativo en variables relacionadas con la infiltración como densidad aparente, porosidad, textura, entre otras. En este sentido Zemke et al. (2019) evaluaron áreas no perturbadas y perturbadas de bosque de pino y encontraron valores de infiltración de 2622 y $935 \mathrm{~mm} \mathrm{~h}^{-1}$, respectivamente, dichas reducciones las atribuyen principalmente a incrementos en la variable de densidad aparente y modificación de la cubierta del suelo.

La cobertura del suelo disminuye la velocidad de escorrentía y afecta directamente la infiltración y el tiempo de escurrimiento superficial (Panachuki et al., 2011). De Almeida et al. (2018) señalan que la infiltración depende directamente del uso de suelo, encontrando que las actividades de labranza tienen un mayor efecto. Específicamente, la infiltración tiende a ser menor en áreas bajo suelo desnudo que en áreas bajo sistemas de conservación, coincidiendo con las tasas de infiltración del uso convencional donde la cobertura del suelo es más baja que en los otros usos agrícolas y el área forestal. 
En el Cuadro 4 se aprecian los valores descriptivos del proceso de infiltración evaluado para cada una de las parcelas. En general el orden de las variables hidrológicas (Fi, Ff, Fb y Fac) para los diferentes usos del suelo fue el siguiente: forestal >macadamia >a. orgánico >a. convencional.

Cuadro 4. Valores descriptivos para las variables hidrológicas evaluadas en las parcelas evaluadas.

\begin{tabular}{|c|c|c|c|c|c|c|}
\hline Variable & Parcelas & Mediana & Varianza & $\mathrm{DE}$ & Mín & Máx \\
\hline \multirow[t]{4}{*}{$\mathrm{Fi}$} & Forestal & 1920 & 15600 & 124.9 & 1740 & 1980 \\
\hline & Aguacate convencional & 600 & 1200 & 34.6 & 600 & 660 \\
\hline & Aguacate orgánico & 840 & 15600 & 124.9 & 660 & 900 \\
\hline & Macadamia & 1200 & 310800 & 557.5 & 1080 & 2100 \\
\hline \multirow[t]{4}{*}{$\mathrm{Ff}$} & Forestal & 891.2 & 5876.7 & 76.7 & 776.8 & 922.4 \\
\hline & Aguacate convencional & 254 & 193.7 & 13.9 & 232.4 & 258.4 \\
\hline & Aguacate orgánico & 407.2 & 1786.7 & 42.3 & 360.8 & 445.2 \\
\hline & Macadamia & 504.4 & 19828.1 & 140.8 & 366.8 & 648.4 \\
\hline \multirow[t]{4}{*}{$\mathrm{Fb}$} & Forestal & 907.5 & 5318 & 72.9 & 804.6 & 945.6 \\
\hline & Aguacate convencional & 260 & 155 & 12.5 & 240.6 & 263.9 \\
\hline & Aguacate orgánico & 419.6 & 2034.5 & 45.1 & 365.8 & 455.5 \\
\hline & Macadamia & 509.1 & 23395.6 & 153 & 372 & 677.3 \\
\hline \multirow[t]{4}{*}{ Fac } & Forestal & 2660.2 & 6914.7 & 26.6 & 262.8 & 309.9 \\
\hline & Aguacate convencional & 868.6 & 2513.3 & 15.5 & 85.5 & 89.6 \\
\hline & Aguacate orgánico & 1311.1 & 1714.3 & 13.4 & 109.7 & 132.1 \\
\hline & Macadamia & 1550.8 & 4732.3 & 68.6 & 119.7 & 252.2 \\
\hline
\end{tabular}

$\mathrm{Fi}=$ infiltración inicial; $\mathrm{Ff}=$ infiltración final; $\mathrm{Fb}=$ infiltración básica; $\mathrm{Fac}=$ infiltración acumulada; $\mathrm{DE}=$ desviación estándar; Min= mínimo; Max= máximo.

Tomé et al. (2015), evaluaron la influencia del cambio de uso de suelo en Andosoles en Tenerife, Islas Canarias, donde compararon con la vegetación natural, encontrando $\mathrm{Fb}$ muy altas fluctuando de 123 a $725 \mathrm{~mm} \mathrm{~h}^{-1}$. En el caso de las áreas con coberturas modificadas disminuyen la $\mathrm{Fb}$ de $98 \mathrm{a}$ $91 \mathrm{~mm} \mathrm{~h}^{-1}$, estos valores a diferencia de los aquí presentados difieren ampliamente en unidades, pero presenta la misma de tendencia de disminución al modificar la vegetación natural y por ende las variables edáficas importantes en procesos hidrológicos como densidad aparente, porosidad y permeabilidad.

La infiltración disminuye conforme pasa el tiempo, durante los primeros minutos la infiltración es rápida, en el minuto 90 comienza a reducirse, donde se aprecia la mayor estabilidad; sin embargo, es notable que el decremento de esta sigue activo en rangos menores, esto puede asociarse al período de la toma de lecturas (Figura 1).

Delgado et al. (2017), mencionan que la intervención de actividades de conservación en áreas degradadas representa una estrategia efectiva para la mejora del recurso suelo-agua-vegetación. Por otra parte, Yáñez-Díaz et al. (2019), evaluaron el efecto del cambio de uso de suelo en un Vertisol en el noreste de México, encontrando tasas de infiltración para un área de matorral 
espinoso tamaulipeco de $226.5 \mathrm{~mm} \mathrm{~h}^{-1}$, pastizal $56.6 \mathrm{~mm} \mathrm{~h}^{-1}$, agricultura $347.9 \mathrm{~mm} \mathrm{~h}^{-1}$ y una plantación de eucaliptus $269.0 \mathrm{~mm} \mathrm{~h}^{-1}$, mencionan que las actividades antropogénicas determinan el funcionamiento del sistema del suelo.

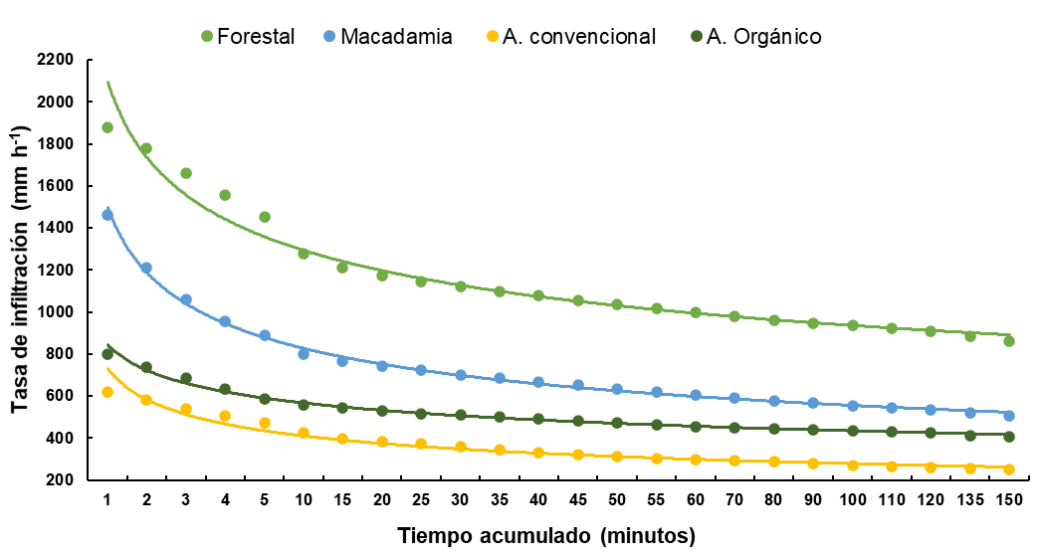

Figura 1. Curvas de infiltración observada en las parcelas evaluadas (a. convencional= aguacate convencional y a. orgánico= aguacate orgánico).

Moro et al. (2005), evaluaron en Argentina la infiltración de un suelo Argiustol údico bajo distintos sistemas de uso, en un monte natural y diferentes sistemas de manejo, observando que la tasa de infiltración disminuye desde el monte natural $324.2 \mathrm{~mm} \mathrm{~h}^{-1}$ a labranza convencional con $90.5 \mathrm{~mm}$ $\mathrm{h}^{-1}$. Leyva et al. (2018) midieron la infiltración en Cuba sobre un Luvisol bajo distintos sistemas de uso arboleda (natural), pastos natural y cultivado y silvopastoril, encontrando una infiltración de 680, 590, 450 y $750 \mathrm{~mm} \mathrm{~h}^{-1}$, respectivamente. Lo anterior, demuestra el impacto negativo que genera el manejo convencional y la conversión de terrenos forestales a sistemas agrícolas sobre cualquier tipo de suelo, disminuyendo la capacidad de la infiltración en los mismos.

\section{Modelos de infiltración}

De los modelos comparados, Kostiakov es el que predice en mejor medida la tasa de infiltración. En general presentó valores para $\mathrm{R}^{2}$ de 0.98 , caso contrario al modelo de Horton que presentó $\mathrm{R}^{2}$ de 0.8 a 0.92 (Cuadro 5). De acuerdo con Haghighi et al. (2010), el modelo de Horton comúnmente presenta los mayores errores en el cálculo de la tasa de infiltración, y Kostiakov es el que mejor se ajusta en suelos con altos contenidos de arena y limo. Zakwan et al. (2016), emplearon diversos modelos para la determinación de la infiltración con la herramienta gradiente reducido generalizado (GRG) de Solver en suelos francos arenosos en Umuahia, Nigeria y documentan que el modelo con el mejor ajuste es Kostiakov basados en los datos obtenidos de campo. Los suelos andosoles generalmente presenta altos contenido de arenas y limos, por lo tanto, el ajuste del modelo de Kostiakov concuerda con lo antes expuesto.

$\mathrm{La} \mathrm{Fb}$ de los datos observados para el uso forestal en la repetición I, II y III fueron de 804.6, 907.5 y $945.6 \mathrm{~mm} \mathrm{~h}^{-1}$, los estimados con Horton presentaron valores de 893.9, 935.2 y $1010.9 \mathrm{~mm} \mathrm{~h}^{-1}$, apreciándose que el modelo sobreestima dichos valores, el modelo de Mezencev emplea variables tanto predictivas, como valores constantes, en este caso el valor de Fb utilizada para el cálculo de la infiltración fue la determinada por los datos observados. 
Cuadro 5. Parámetros de infiltración obtenidos a partir de los modelos.

\begin{tabular}{|c|c|c|c|c|c|c|c|c|c|c|c|c|}
\hline \multirow{2}{*}{ Parcelas } & \multirow{2}{*}{ Repetición } & \multicolumn{4}{|c|}{ Horton } & \multicolumn{3}{|c|}{ Kostiakov } & \multicolumn{4}{|c|}{ Mezencev } \\
\hline & & $\mathrm{Fb}$ & F0 & $\mathrm{K}$ & $\mathrm{R}^{2}$ & $\mathrm{a}$ & $\mathrm{b}$ & $\mathrm{R}^{2}$ & $\mathrm{Fb}$ & $\mathrm{a}$ & $b$ & $\mathrm{R}^{2}$ \\
\hline \multirow[t]{3}{*}{ Forestal } & I & 893.9 & 1904.4 & -0.09 & 0.9 & 1971.2 & -0.18 & 0.98 & 804.6 & 1293.7 & $7-0.51$ & 0.94 \\
\hline & II & 935.2 & 1775.8 & -0.11 & 0.88 & 1748.7 & -0.15 & 0.98 & 907.5 & 980.3 & -0.6 & 0.91 \\
\hline & III & 1010.9 & 1838 & -0.04 & 0.91 & 2036.4 & -0.14 & 0.98 & 945.6 & 1180.2 & -0.42 & 0.96 \\
\hline \multirow{3}{*}{$\begin{array}{c}\text { Aguacate } \\
\text { Convencional }\end{array}$} & I & 301.1 & 553.6 & -0.08 & 0.9 & 579.5 & -0.15 & 0.98 & 263.8 & 354.7 & -0.47 & 0.95 \\
\hline & II & 250.8 & 586.1 & -0.03 & 0.91 & 667.5 & -0.19 & 0.98 & 240.6 & 445.9 & -0.42 & 0.95 \\
\hline & III & 258.6 & 627 & -0.06 & 0.91 & 693.7 & -0.21 & 0.98 & 260 & 490.4 & -0.48 & 0.94 \\
\hline \multirow[t]{3}{*}{ Aguacate Orgánico } & I & 378.6 & 610.3 & -0.04 & 0.92 & 658.6 & -0.12 & 0.98 & 365.8 & 323.3 & -0.45 & 0.95 \\
\hline & II & 504.7 & 939.3 & -0.25 & 0.83 & 800.7 & -0.12 & 0.98 & 455.4 & 411.5 & -0.53 & 0.93 \\
\hline & III & 453.6 & 848.7 & -0.07 & 0.9 & 891.5 & -0.16 & 0.98 & 419.6 & 534.4 & -0.51 & 0.94 \\
\hline \multirow[t]{3}{*}{ Macadamia } & I & 778.2 & 1770.3 & -0.05 & 0.92 & 1999.5 & -0.21 & 0.98 & 677.3 & 1454.8 & $3-0.48$ & 0.94 \\
\hline & II & 412.3 & 1226.2 & -0.26 & 0.82 & 987.7 & -0.22 & 0.98 & 371.9 & 741.8 & -0.68 & 0.89 \\
\hline & III & 569.9 & 1475.7 & -0.35 & 0.8 & 1091.7 & -0.17 & 0.98 & 509.1 & 720.1 & -0.63 & 0.9 \\
\hline
\end{tabular}

$\mathrm{Fb}=$ infiltración básica, $\mathrm{K}$, a, b y F0 = parámetros determinados por los modelos (donde: F0 y a representan la infiltración inicial determinada por el modelo); $\mathrm{R}^{2}=$ coeficiente de determinación.

De acuerdo con los resultados, los tres modelos pueden predecir el proceso de infiltración, ajustándose de manera significativa; sin embargo, en el modelo de Kostiakov sobresale el estadístico del coeficiente de determinación $\left(\mathrm{R}^{2}\right)$ en todas las pruebas de infiltración, con valores cercanos a 1. Es notable que el modelo de Kostiakov es el que predice una mejor tasa de infiltración, ya que representa los valores más aproximados a la infiltración real, mientras tanto Horton y Mezencev sobreestiman dichos valores. Lo anterior coincide con Luna et al. (2020), quienes ajustaron los mismos modelos a un suelo tipo Umbrisol sometido a manejo forestal, obteniendo coeficientes de determinación similares a las del presente estudio. Diversos autores emplean indicadores estadísticos como objeto de validación de modelos de simulación para la evaluación de la infiltración (Alega et al., 2016; Sihag y Singh, 2018; Suryoputro et al., 2018).

Los índices NSE y r alcanzaron valores superiores a 0.9 para todos los modelos al igual que usos de suelo, mientras que el MAE fue alto para todos los modelos, indicando un buen ajuste de los datos observados respecto a los simulados. El rango en el que se encuentra el estadístico NSE es considerado como óptimo de acuerdo con Zakwan et al. (2016). Por otra parte, el modelo de Kostiakov presentó los NSE y r más elevados y el menor MAE lo cual lo hace el mejor modelo de predicción para la infiltración de agua en suelos Andosoles (Cuadro 6).

Alega et al. (2016) mencionan que los índices estadísticos permiten evaluar la exactitud de los modelos de infiltración. Suryoputro et al. (2018) evaluaron la eficiencia del ajuste de modelos de infiltración en distintos usos de suelo: asentamientos, plantación, cultivo de arroz y bosque, encontrando un NSE de 0.68, 0.73, 0.91 y 0.49, respectivamente, empleando los modelos Kostiakov, Kostiakov-Lewis y Green-Ampt, con $\mathrm{R}^{2}$ de 0.52 a 0.94. 
Cuadro 6. Valores medios de los indicadores estadísticos de los modelos en las parcelas evaluadas.

\begin{tabular}{cccccc}
\hline \multirow{2}{*}{ Modelos } & \multirow{2}{*}{ Variable } & \multicolumn{4}{c}{ Parcelas } \\
\cline { 3 - 6 } Horton & NSE & 0.95 & A. convencional & A. orgánico & Macadamia \\
\hline \multirow{3}{*}{ Kostiakov } & $\mathrm{r}$ & 0.98 & 0.95 & 0.93 & 0.92 \\
& $\mathrm{MAE}$ & 50.67 & 17 & 0.96 & 0.96 \\
& $\mathrm{NSE}$ & 0.98 & 0.98 & 22.38 & 52.99 \\
& $\mathrm{r}$ & 0.99 & 0.99 & 0.97 & 0.95 \\
Mezencev & $\mathrm{MAE}$ & 32.73 & 12.75 & 0.99 & 0.98 \\
& $\mathrm{NSE}$ & 0.93 & 0.91 & 11.09 & 33.77 \\
& $\mathrm{r}$ & 0.97 & 0.96 & 0.94 & 0.97 \\
& $\mathrm{MAE}$ & 61.92 & 27.32 & 0.97 & 0.99 \\
\hline
\end{tabular}

$\mathrm{NSE}=$ coeficiente de eficiencia del modelo Nash Sutcliffe; $r=$ coeficiente de Pearson y MAE= error absoluto medio.

En general, los resultados del presente estudio arrojaron estadísticos que permiten establecer la eficacia de los modelos para la predicción de la infiltración aplicados al suelo tipo andosol. Sihag y Singh (2018) determinaron que el modelo de Kostiakov presenta el mejor ajuste y NSE (0.99), coincidiendo con los valores del presente estudio. Por otra parte, Bayabil et al. (2019), encontraron que los modelos de Horton y Kostiakov predicen de manera más eficiente la infiltración hallando valores de ajuste de $\mathrm{R}^{2}$ y NSE de 0.97 , comparado con la presente investigación, los valores obtenidos son similares.

\section{Conclusiones}

Los cambios de uso de suelo forestal a usos agrícolas provocaron cambios en las variables hidrológicas del recurso suelo. Dichos cambios se encuentran influenciados con las distintas prácticas agrícolas empleadas y las características edáficas de las parcelas evaluadas. En este sentido, las clases de textura para las dos parcelas orgánicas y uso forestal fueron franco arenoso, mientras que el uso convencional presentó una clase franco limoso siendo particularmente el área donde se presentó una infiltración baja.

Las variables hidrológicas del proceso de infiltración ( $\mathrm{Fi}, \mathrm{Ff}, \mathrm{Fb}$ y Fac) presentaron el mismo orden decreciente siguiente: forestal> macadamia> aguacate orgánico> aguacate convencional. Es importante señalar que el cambio de uso de suelo de cubierta forestal a cultivos agrícolas genera impactos negativos en la infiltración, pero la aplicación de actividades conservacionistas, como el manejo orgánico que representa una alternativa para la mitigación de dicho impacto en la infiltración de agua en el suelo, regulando la interacción suelo-agua-vegetación.

De acuerdo con los resultados obtenidos por los indicadores estadísticos (NSE, r, MAE y R²), el modelo de Kostiakov es altamente eficiente para describir el comportamiento de la infiltración en suelos Andosoles. Sin embargo, Horton y Mezencev también describen adecuadamente dichos parámetros. Los modelos de infiltración pueden ser considerados como una herramienta para la toma de decisiones, ya que los modelos cumplen con un grado de ajuste a los datos reales y pueden ser empleados para la predicción de la infiltración en suelos Andosoles bajo distintas coberturas. 


\section{Literatura citada}

Alcalá, M. J.; Ortiz, C. A. y Gutiérrez, M. D. C. 2001. Clasificación de los suelos de la meseta tarasca, Michoacán. Terra Latinoam. 19(3):227-239.

Alega, A. M. G. 2016. Evaluación de tres modelos en la estimación de la infiltración acumulada. Rev. Ingen. Agríc. 6(3):41-47.

Batres, C. A. y Barahona-Palomo, M. 2016. Comparación de tres métodos de infiltración para calcular el balance hídrico del suelo, en la cuenca del río suquiapa, El salvador. UNED research journal/cuadernos de investigación UNED. 9(1):23-33.

Bayabil, H. K.; Dile, Y. T.; Tebebu, T. Y.; Engda, T. A. and Steenhuis, T. S. 2019. Evaluating infiltration models and pedotransfer functions: implications for hydrologic modeling. Geoderma. 338:159-169. https://doi.org/10.1016/j.geoderma.2018.11.028.

Bejar, P. S. J.; Cantú, S. I.; Yáñez, D. M. I. y Luna, R. E. O. 2020. Curvas de retención de humedad y modelos de pedotransferencia en un Andosol bajo distintos usos de suelo. Rev. Mex. Cienc. Forest. 11(59):31-50. https://doi.org/10.29298/rmcf.v11i59.666.

Bravo-Espinosa, M.; Mendoza, M. E.; Carlón Allende, T.; Medina, L.; Sáenz-Reyes, J. T. and Páez, R. 2014. Effects of converting forest to avocado orchards on topsoil properties in the transMexican volcanic system, Mexico. Land Degradation \& Development. 25(5):452-467. https://doi.org/10.1002/ldr.2163.

Burgos, A.; Anaya, C. y Cuevas, G. 2012. Impacto ecológico del Cultivo de Aguacate a nivel regional y de parcela en el estado de Michoacán: definición de una tipología de productores, etapa II. Informe final, Fundación Produce Michoacán. Centro de Investigaciones en Geografía Ambiental- Universidad Nacional Autónoma de México (UNAM). Morelia, Michoacán, $125 \mathrm{p}$.

Castellanos-Navarrete, A.; Chocobar-Guerra, A.; Cox, R.; Fonteyne, S.; Govaerts, B.; Jespers, N. y Verhulst, N. 2013. Infiltración: guía útil para comparar las prácticas de manejo de cultivo. Centro Internacional del Maíz y Trigo (CIMMYT). México, DF. 8 p.

Chávez, L. G.; Tapia, V. L. M.; Bravo, E. M.; Sáenz, R. J.; Muñoz, F. H. J.; Vidales F. I. y Mendoza C. M. 2012. Impacto de cambio de uso de suelo forestal a huertos de aguacate. (Ed.). Instituto Nacional de Investigaciones Forestales, Agrícolas y Pecuarias (INIFAP)- Centro de Investigación Pacífico Centro-Campo Experimental Uruapan. Libro técnico núm. 13. 76 p.

De Almeida, W. S.; Panachuki, E.; De-Oliveira, P. T. S.; Da-Silva, M. R.; Sobrinho, T. A. and de Carvalho, D. F. 2018. Effect of soil tillage and vegetal cover on soil water infiltration. Soil Tillage Res. 175:130-138. http://dx.doi.org/10.1016/j.still.2017.07.009.

Delgadillo, O. y Pérez, L. 2016. Medición de la infiltración del agua en el suelo. Centro andino para la gestión y uso del agua. Centro agua. Facultad de ciencias agrícolas, pecuarias y forestales universidad mayor de san simón. Cochabamba, Bolivia. 31 p.

Delgado, M. I.; Gaspari, F. J. y Senisterra, G. E. 2017. Respuesta a la infiltración en distintos complejos suelo-vegetación en las sierras de Ventania, Argentina. Rev. Tecnología. 16(1):157-168. http://hdl.handle.net/11336/56680.

Florez, F. H.; Triana, F. A. y Torrez, M. C. D. 2009. Efecto de actividades agropecuarias en la capacidad de infiltración de los suelos del páramo del sumapaz. Ingeniería de Recursos Naturales y del Ambiente. 8:29-38. https://www.redalyc.org/pdf/2311/ 231116390004.pdf.

Galicia, L. 2014. El cambio de uso de suelo: consecuencias en el ciclo hidrológico y la disponibilidad de agua. AZ. Rev. Educ. Cult. 82:15-18. 
García-Hernández, M. A.; García-Hernández, M. A.; Castellano-Vargas, I.; Cano-Santana, Z. y Peláez-Rocha, C. M. 2008. Variación de la velocidad de infiltración media en seis ecosistemas inalterados. Terra Latinoam. 26(1):21-27.

Green, W. H. and Ampt, G. A. 1911. Studies on Soil Phyics. The J. Agric. Sci. 4(1):1-24. https://doi.org/10.1017/S0021859600001441

Haghighi, F.; Gorji, M.; Shorafa, M.; Sarmadian, F. and Mohammadi, M. H. 2010. Evaluation of some infiltration models and hydraulic parameters. Spanish J. Agric. Res. 8(1):210-217.

Horton, R. E. 1939. Analysis of runoff-plot experiments with varying infiltration capacity, transactions American Goephysical union, Washington. 693-711.

Landini, A. M.; Martínez, D. A.; Días, H.; Soza, E.; Agnes, D. y Sainato, C. M. 2007. Modelos de infiltración y funciones de pedotransferencia aplicados a suelos de distinta textura. Rev. Cienc. Suelo. 25(2):123-131.

Leyva, R.; Laura, S.; Baldoquín, P. y Reyes, O. 2018. Propiedades de los suelos en diferentes usos agropecuarios, las tunas, Cuba. Rev. Cienc. Agríc. 35(1):36-47. http://dx.doi.org/10.22267/ rcia.183501.81.

Luna, R. E. O.; Cantú, S. I.; Yáñez, D. M. I.; González, R. H.; Marmolejo, M. J. G. y Béjar P. S. J. 2020. Ajuste de modelos empíricos de infiltración en un umbrisol bajo diferentes tratamientos silvícolas. Rev. Mex. Cienc. Forest. 11(57):132-152. https://doi.org/10.29298/ rmcf.v11i57.643.

Mishra, S. K.; Tyagi, J. V. and Singh, V. P. 2003. Comparison of infiltration models. Hydrological Processes. 17(13):2629-2652. https://doi.org/10.1002/hyp.1257.

Moro, E. C.; Ingaramo, O. E.; Venialgo, C. A. y Gutiérrez, N. C. 2005. Infiltración en un argiustol undico con diferentes sistemas de uso en el departamento 9 de Julio-Chaco. Agrotecnia. 14:14-18.

Panachuki, I.; Bertol, T.; Alves, P. T. S.; Oliveira, D. B. B. y Rodrigues, B. 2011. Perdas de solo e de água e infiltração de água em latossolo vermelho sob sistemas de manejo. Rev. Bras. Ciênc. Solo. 35(5):1777-1785. http://dx.doi.org/10.1590/S0100-06832011000500032.

Regüés, D.; Serrano-Muela, P.; Nadal-Romero, E. y Lana-Renault, N. 2012. Análisis de la variabilidad temporal de la infiltración en un gradiente de degradación de usos del suelo en el pirineo central. Cuaternario y Geomorfología. 26(1-2):9-28.

Rivera, J. S. Ú. y Dallatorre, Y. D. 2018. La infiltración del agua en los suelos y componentes artificiales y materia orgánica que se utilizan en ellos para la agricultura. Rev. Iberoamérica. Bioeconomía y Cambio Climático. 4(7):889-896. http://dx.doi.org/10.5377/ ribcc.v4i7.6299.

Rzedowski, J. 2006. Vegetación de México. $1^{\text {a }}$ Edición, Limusa (Ed). Limusa. 382 p.

Sihag, P. and Singh B. 2018. Field evaluation of infiltration models. Sci. Technical J. 4(2):3-12. http://doi.org/10.5281/zenodo.1239447.

Singh, B.; Sihag, P. and Singh, K. 2018. Comparison of infiltration models in NIT Kurukshetra campus. Appl. Water Sci. 8(2):63-70. https://doi.org/10.1007/s13201-018-0708-8.

Suryoputro, N.; Soetopo, W.; Suhartanto, E. S. and Limantara, L. M. 2018. Evaluation of infiltration models for mineral soils with different land uses in the tropics. J. Water Land Development. 37(1):153-160. https://doi.org/10.2478/jwld-2018-0034.

Tejedor, M.; Neris, J. y Jiménez, C. 2013. Propiedades del suelo que controlan la infiltración en suelos volcánicos (Tenerife, España). Rev. Socied. Cienc. Suel. América. 77(1):202-212. https://doi.org/10.2136/sssaj2012.0132. 
Tomé, J. N.; Cerezal, J. C. S.; Prieto, F. P.; Pérez, J. A. y Villegas, P. G. 2015. Hidrología, erosión y restauración de suelos volcánicos afectados por incendios forestales: experiencia en las islas canarias. In: restauración de la cubierta vegetal y de espacios degradados en la región de macaronesia. Colegio de Ingenieros de Montes. Madrid, España. 279-294 pp.

Weber, J. y Apestegui, L. 2013. Parámetros del modelo de infiltración de green y ampt en suelos de la ciudad de Córdoba, Argentina. Cuadernos del CURIHAM. 19:87-103.

Weber, J. F. y Apestegui, L. 2016. Relaciones entre parámetros de los modelos de infiltración de Kostiakov y Lewis-Kostiakov-Córdoba, Argentina. Tecnología y Ciencias del Agua. 7(2):115-132.

Yáñez-Díaz, M. I.; Cantú-Silva, I.; González-Rodríguez, H. and Sánchez-Castillo, L. 2019. Effects of land use change and seasonal variation in the hydrophysical properties in vertisols in northeastern Mexico. Soil Use and Management. 35(3):378-387. https://doi.org/10.1111/ sum. 12500 .

Zakwan, M.; Muzzammil, M. and Alam, J. 2016. Application of spreadsheet to estimate infiltration parameters. Perspec. Sci. 8:702-704. https://doi.org/10.1016/j.pisc.2016.06.064.

Zhang, J.; Lei, T.; Qu, L.; Chen, P.; Gao, X.; Chen, C. and Su, G. 2017. Method to measure soil matrix infiltration in forest soil. J. Hydrol. 552:241-248. http://dx.doi.org/10.1016/ j.jhydrol.2017.06.032.

Zemke, J. J.; Enderling, M.; Klein, A. and Skubski, M. 2019. The influence of soil compaction on run of formation. A case study focusing on skid trails at forested andosol sites. Geosciences. 9(204):2-14. https://doi.org/10.3390/geosciences9050204. 